\title{
Jeux de rôle pour médecins-chefs et chefs de clinique
}

\author{
Lors d'un atelier pilote organisé à Lucerne, des médecins-cadres ont été formés à la \\ manière de mieux accompagner et encadrer leurs collaborateurs. Cet atelier conçu \\ par l'Institut suisse pour la formation médicale postgraduée et continue (ISFM) en \\ collaboration avec le Royal College of Physicians de Londres a été extrêmement bien \\ accueilli par les participants, si bien que des cours de ce type seront désormais \\ régulièrement proposés.
}

La jeune médecin-assistante fond tout à coup en larmes. Debout devant la chambre du patient, elle rougit de honte alors que son chef lui pose une question après l'autre; ce qui ressemble plus à un interrogatoire devant un tribunal. Une infirmière arrive à grand pas, reflet du stress quotidien d'un service hospitalier. Est-elle consciente de l'erreur qu'elle a commise, tempête le médecin-chef. «Si, bien sûr, mais...» La jeune praticienne n'arrive pas à terminer sa phrase. Et pourquoi avait-elle fait ça? poursuit le médecin hors de lui. La jeune femme essaie de s'expliquer en balbutiant. Mais le médecin l'écoute à peine et lâche en s'éloignant: «il va y avoir des conséquences.» Hors d'elle, la jeune femme le regarde s'éloigner.

\section{Intérêt marqué pour les cours}

Son désespoir est palpable, mais par chance tout n'est que fiction. La jeune femme et l'homme aux cheveux gris jouent le rôle d'un médecin-assistant et d'un médecin-chef dans un hôpital en Grande-Bretagne. Le tout est un clip vidéo, suivi avec attention par les 35 médecins présents à la première journée d'une formation composée de plusieurs ateliers. Les médecins en charge de la formation apprennent à mieux diriger leurs collaborateurs, à régler le mieux possible les problèmes et les conflits et tout ce qui contribue à la cohésion de l'équipe. Cette formation s'inscrit dans un projet pilote, initié et organisé par l'Institut suisse pour la formation médicale postgraduée et continue (ISFM) en collaboration avec le Royal College of Physicians de Londres. «Les médecins dirigeants rencontrent toujours plus de difficultés à assumer leur rôle», déclare Werner Bauer, président de l'ISFM. Cela tient d'abord à la bureaucratie croissante qui réduit le temps dont ils disposent. «En outre, beaucoup ont du mal à assumer leur fonction dirigeante face à de jeunes collègues qui les remettent en question.» Lors d'un déplacement au Royal College il y a deux ans, Werner Bauer a été conquis par les formations proposées par cette institution. «Je me suis dit que c'était vraiment cela dont nos médecins avaient besoin!» Il se met alors rapidement d'accord sur le contenu et le déroulement avec les organisateurs des ateliers et, une année plus tard, les inscriptions étaient ouvertes. «L'intérêt dépassa toutes les espérances: en quelques jours, le cours était plein», raconte Werner Bauer. L'atelier comprenait deux cours d'un jour et un cours de deux jours. Dans les deux premiers cours, les participants ont découvert des stratégies efficaces pour enseigner le mieux possible et pour assurer le meilleur soutien possible à des collaborateurs présentant un faible niveau de performance. Le troisième cours, celui de deux jours, a été centré sur les qualités dirigeantes, notamment le feed-back constructif, la bonne gestion des collaborateurs à faible rendement et le développement des propres aptitudes à diriger.

\section{Solutions concrètes aux problèmes du quotidien}

«D'après vous, qu'est-ce qui n'allait pas?», s'est enquis David Perry lors d'un premier tour de table auprès des participants après le premier film. D'abord par deux, puis dans un plus grand groupe, les participants ont eu des échanges nourris sur le comportement du médecin-chef britannique. «Je voulais apprendre comment et dans quel environnement je pouvais mener des entretiens de qualification", commente Frank Zimmermann, médecin-chef du service de radio-oncologie à l'Hôpital universitaire de Bâle. «Le cours m'a ici réellement aidé.» De nombreux exemples concrets et pratiques ont été apportés et discutés. "Il nous a été constamment suggéré de nous mettre dans le rôle d'un autre et d'essayer de résoudre ensemble les problèmes - c'était vraiment bien.» Seul bémol, le temps imparti aux jeux de rôle était parfois trop court. «Je ne peux que vivement recommander ce cours - le meilleur moment de le suivre est sans doute la dernière année avant l'examen de spécialiste ou avant l'entrée en fonction dans une position dirigeante.» F. Zimmermann prévoit de rendre cet atelier obligatoire pour ses collaborateurs. Même après le cours, Martin Beck, médecin-chef en orthopédie à l'Hôpital cantonal de Lucerne, trouve toujours pénible de communiquer un feed-back à des collaborateurs problématiques. «Mais je peux maintenant m'appuyer sur des techniques avec les- 


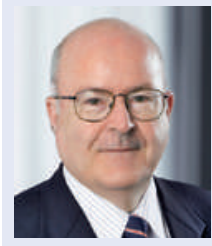

Interview avec le Dr Werner Bauer, président de l'Institut suisse pour la formation médicale postgraduée et continue (ISFM)

BMS: Les médecins dirigeants ont-ils besoin d'un cours pour apprendre à diriger leurs collaborateurs?

Dr Werner Bauer: Besoin au sens d'une obligation, évidemment pas. En revanche, pour de jeunes chefs de clinique, il n'est pas facile aujourd'hui d'assumer leur rôle de supérieur hiérarchique. Les hiérarchies se sont beaucoup aplanies et les jeunes médecins remettent davantage en question l'autorité des plus expérimentés qu'auparavant. Les jeunes chefs de clinique doivent d'abord s'accoutumer à leur position de dirigeant; sur ce point, le cours peut leur être très bénéfique. Ainsi qu'aux médecins plus âgés qui peuvent en tirer un profit considérable.

\section{BMS: Comment l'idée vous est-elle venue?}

W. Bauer: Nous constatons que les médecins-cadres disposent de moins en moins de temps au quotidien pour s'occuper de la formation postgraduée de leurs jeunes collègues. Les tâches administratives ne cessent d'augmenter. Comment peut-on dans ce cas trouver encore du temps pour penser à la meilleure manière d'encadrer ses jeunes médecins-assistants? A I'ISFM, nous sommes donc parvenus à la conclusion suivante: nous voulions proposer quelque chose qui aide les médecins dirigeants et les médecins-chefs à mieux assumer leur mission d'enseignement et de direction.

BMS: Comment le cours a-t-il vu concrètement le jour?

W. Bauer: En tant que président de l'Association européenne des internistes, je suis devenu Fellow du Royal College of Physicians of London en
2010. A ce titre, j'ai eu l'occasion de visiter le département de l'éducation médicale. J'ai découvert que les organisateurs proposaient des cours sur le thème «Teach the Teacher» dans le monde entier et j'ai trouvé l'idée convaincante: c'était exactement ce que nous recherchions. Pourquoi réinventer un cours alors que le Royal College possédait déjà des années d'expérience?

BMS: Avez-vous laissé carte blanche aux organisateurs concernant le contenu et la structure du cours ou y avez-vous pris part?

W. Bauer: Nous avons planifié ensemble les ateliers. L'ISFM a défini les thèmes qui lui tenaient à cœur et les experts du Royal College ont ensuite adapté le cours à nos besoins.

BMS: Le premier cours a été un véritable succès (voir article). Des ateliers vont-ils désormais avoir lieu régulièrement?

W. Bauer: Oui, nous sommes déjà en train de planifier les prochains cours, qui se tiendront également à Lucerne. Pour l'aménagement des contenus, nous étudierons attentivement les retours d'information des participants et adapterons les cours aux propositions et souhaits émis. Nous souhaitons par exemple préciser à l'avance les thèmes des différentes journées.

BMS: Le premier cours n'a coûté qu'une taxe administrative de 50 francs, le reste ayant été pris en charge par I'ISFM. Ce système sera-t-il maintenu? W. Bauer: Ce n'était malheureusement possible que pour les cours pilotes. Nous prévoyons un coût approximatif de 250 francs pour une journée et de 450 francs pour deux jours. Mais nous nous efforçons de limiter les coûts. Nous souhaitons proposer aux médecins chargés de la formation postgraduée en Suisse un cours d'excellente qualité à un prix raisonnable les formateurs contribuent en effet énormément à la formation de la relève médicale. quelles je parviens à dédramatiser la situation ou à éviter une escalade.» Martin Beck estime que le cours lui a apporté des clés pour résoudre les problèmes du quotidien et que l'échange avec les autres participants a été très constructif. Mais c'est principalement pour améliorer sa manière d'enseigner qu'il a suivi ce cours. "Ceux qui vont prendre la relève doivent être très bien formés», souligne notre interlocuteur, «Bien enseigner, ça s'apprend.»

\section{Les formateurs ont aussi besoin de soutien}

«Avec nos ateliers, nous souhaitons donner aux médecins un savoir et des compétences pour le domaine non clinique», déclare Winnie Wade, coorganisatrice de l'atelier et directrice de la formation au Royal College of Physicians de Londres. L'ensemble des ateliers sont dispensés conjointement par des médecins et des pédagogues expérimentés ce qui est «la clé du succès de nos programmes», explique-t-elle. Les pédagogues transmettent le savoir théorique et des conseils pratiques; les médecins apportent leur expérience et des connaissances tirées de la pratique médicale. Le Royal College a lancé ces ateliers il y a plus de douze ans maintenant. «A cette époque, il existait peu de choses pour préparer les médecins à leur tâche de direction», explique Winnie Wade. Puis, dans les années qui ont suivi, les cours ont été adaptés aux besoins des médecins et développés dans ce sens. Ils sont désormais proposés dans le monde en- tier, en particulier en Europe, aux USA et en Asie du Sud. En Suisse, l'enthousiasme et l'intérêt des médecins pour ce cours se sont manifestés par une participation très active aux exercices. Elle espère que le partenariat avec l'ISFM sera de longue durée et que les cours apporteront une aide à de nombreux médecins dans leur évolution professionnelle.

«Aujourd'hui, beaucoup de médecins accèdent à la fonction de chefs de clinique alors qu'ils sont encore relativement jeunes», constate Simon Ritter, médecin-chef avec une responsabilité élargie en médecine interne à l'Hôpital Triemli à Zurich; «tout sformateur en formation postgraduées a encore besoin de soutien, et il doit diriger et former correctement les médecins-assistants.» Malheureusement, le quotidien du médecin ne laisse que trop peu de temps pour cette tâche. «Les cinq étapes du modèle Mikroskill, que nous avons découvert au cours, sont une grande aide pour moi», affirme Simon Ritter. Selon ce modèle, l'étudiant en médecine ou le médecin-assistant est

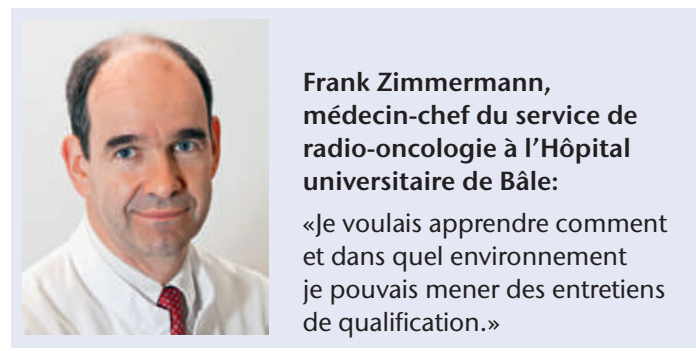




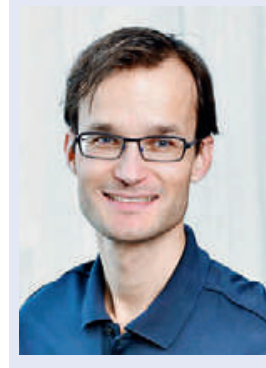

Simon Ritter, médecin-chef avec une responsabilité élargie en médecine interne à l'Hôpital Triemli à Zurich:

«Tout «formateur en formation postgraduées a encore besoin de soutien, et il doit diriger et former correctement les médecinsassistants.»

appelé à se déterminer sur un diagnostic ou sur un plan de traitement et à justifier son choix. Puis les principaux aspects de la maladie lui sont expliqués. A la fin, on félicite le jeune médecin pour tous les actes corrects et l'on corrige les fautes commises. Pour ce dernier aspect, la critique est formulée de manière constructive avec des propositions d'amélioration concrètes. "Cela permet de renforcer une réflexion ciblée et critique chez les étudiants et médecins-assistants, et de les familiariser avec la prise de décision dans le quotidien hospitalier», résume S. Ritter. Par ailleurs, il reconnait avoir mieux compris la dynamique d'un petit groupe d'apprenants. «Lors de mes cours pour étudiants, je peux beaucoup mieux m'adapter aux besoins des praticiens en formation au lieu de m'appuyer sur un concept rigide pour l'enseignement.»

Le cours a été entièrement donné en anglais, ce qui n'a pas présenté de problème particulier pour la plupart des participants. «J'aurais néanmoins préféré que le cours soit dispensé en allemand», déclare Ueli Zellweger, médecin-chef de la division médicale à l'Hôpital régional de la Surselva. «Et même si j'ai tout compris, la petite pointe d'humour ou la subtilité de sens dans un dialogue m'a parfois échappé.» Mais, en

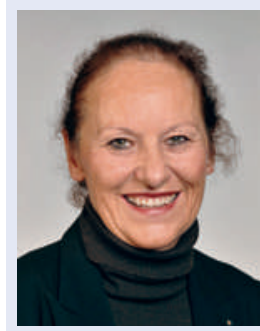

Elisabeth Bandi-Ott, responsable d'enseignement à l'Institut de médecine de famille à l'université de Zurich:

«Dans une logique d'assurance qualité, les médecins-assistants doivent pouvoir être bien formés après l'obtention de leur diplôme de médecin.»

dépit de cette barrière de la langue, Ueli Zellweger relève l'ensemble des conseils et des aides qu'il pourra utiliser à l'avenir. «Je trouve par exemple intéressante la proposition de faire régulièrement et systématiquement évaluer les médecins-assistants par des collaborateurs non-médecins tels les soignants ou les physiothérapeutes», déclare Ueli Zellweger. Dans un petit hôpital régional, la formation des médecins n'est pas toujours aisée. «Le défi subsistera toujours de communiquer à un médecin-assistant que ses prestations sont insuffisantes, tout en le motivant et en l'incitant à s'améliorer et à augmenter la qualité de ses prestations», souligne Ueli Zellweger. «Cependant, j'ai remarqué combien il était nécessaire de mener régulièrement des entretiens de feed-back.»

\section{Souhaits pour les cours à venir}

D'un point de vue objectif également, les ateliers ont connu un grand succès comme le montre l'évaluation des questionnaires. La plupart des participants ont jugé bonne voire très bonne la structure des cours qui leur ont donné largement satisfaction et qu'ils ont suivis avec plaisir. D'autre part, les participants ont estimé que les cours correspondaient largement ou même très largement à ce qui était annoncé et apportaient un contenu pertinent pour la pratique. Il existe désormais une longue liste de souhaits pour les cours à venir: les participants aimeraient par exemple savoir comment mieux diriger les médecins-assistants trop sûrs d'eux, comment mieux gérer et répartir le temps à disposition et comment aborder les situations de crise. «Les réactions ont été à ce point positives que le cours sera à nouveau pro-

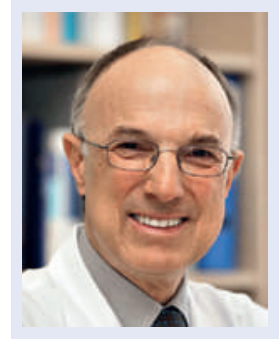

Ueli Zellweger, médecin-chef de la division médicale à l'Hôpital régional de la Surselva:

«Cependant, j'ai remarqué combien il était nécessaire de mener régulièrement des entretiens de feed-back.»

posé l'année prochaine», ajoute W. Bauer. Malheureusement, son coût va nettement excéder les 50 francs de frais de traitement de dossier d'inscription. «J'aurais quand même suivi le cours», indique Elisabeth Bandi-Ott, responsable d'enseignement à l'Institut de médecine de famille à l'université de Zurich. «Dans une logique d'assurance qualité, les médecins-assistants doivent pouvoir être bien formés après l'obtention de leur diplôme de médecin», insiste-t-elle. Dans ce contexte, le cours apporte les bonnes réponses: «un feed-back constructif est l'un des éléments essentiels de la formation.»

Dans une deuxième séquence vidéo, les participants peuvent découvrir la bonne méthode suivie par le médecin-chef britannique: il convie le médecin-assistant à un entretien dans une pièce tranquille et demande qu'ils ne soient pas dérangés. Avec calme, il écoute ce qu'elle a à dire et pose des questions au moment opportun. Il apprend ainsi que la jeune femme se sent très seule dans cette ville et qu'elle souffre, en plus, de graves insomnies. Les nombreuses gardes de nuit la fatiguent énormément. Pour elle, l'erreur commise est certainement à mettre en relation avec ces circonstances difficiles. Le chef lui recommande alors de chercher un médecin de famille et convient avec elle d'une réduction de ses horaires. Il revient également sur la faute commise et réexplique quelles auraient pu en être les conséquences. Soulagée mais aussi satisfaite, le médecin-assistant promet de se mettre en quête d'un médecin de famille et s'engage à l'avenir de tout mettre en œuvre pour éviter qu'une telle erreur ne se reproduise. 